\title{
Multiple Solutions for a Sixth Order Boundary Value Problem
}

\author{
A. L. M. MARTINEZ ${ }^{*}$, C. A. PENDEZA MARTINEZ ${ }^{2}$, G. M. BRESSAN ${ }^{3}$, \\ R. MOLINA SOUZA ${ }^{4}$ and E. W. STIEGELMEIER ${ }^{5}$
}

Received on April 11, 2020 / Accepted on November 18, 2020

\begin{abstract}
This work presents conditions for the existence of multiple solutions for a sixth order equation with homogeneous boundary conditions using Avery Peterson's theorem. In addition, non-trivial examples are presented and a new numerical method based on the Banach's Contraction Principle is introduced.
\end{abstract}

Keywords: numerical solutions, sixth-order, boundary value problem and multiple solutions

In this manuscript we address conditions for the existence of multiple solutions for the sixth order limit value problem:

$$
\begin{gathered}
u^{(6)}+f(t, u)=0, \quad 0<t<1, \\
u(0)=u^{\prime}(0)=u^{\prime \prime}(0)=0, u^{\prime}(1)=u^{\prime \prime \prime}(1)=u^{(5)}(1)=0 .
\end{gathered}
$$

where $f: R^{2} \rightarrow R$ is a continue function.

In the literature, there are several studies mainly focused only on the existence of solutions with qualitative and quantitative aspects. Among them, we recommend [1], [2], [3], [5], [13], [6], [7], [8], [12], [4] and the references therein.

Some specific studies, as [5], [8] and [14], have analyzed conditions for the existence of solutions for this class of problems. In [14], the authors approach a simplified version of problem, in which they consider the dependence of $f$ only on $t$, the authors apply the Krasnoselskii's fixed point theorem to determine sufficient conditions for the existence of a positive solution.

\footnotetext{
*Corresponding author: André Luís Machado Martinez - E-mail: martinez@utfpr.edu.br

${ }^{1}$ Departamento Acadêmico de Matemática, Universidade Tecnológica Federal do Paraná, Cornélio Procópio, Paraná, Brazil - E-mail: martinez@utfpr.edu.br https://orcid.org/0000-0003-1888-648X

2 Departamento Acadêmico de Matemática, Universidade Tecnológica Federal do Paraná, Cornélio Procópio, Paraná, Brazil - E-mail: crismartinez@utfpr.edu.br https://orcid.org/0000-0003-3891-744X

3 Departamento Acadêmico de Matemática, Universidade Tecnológica Federal do Paraná, Cornélio Procópio, Paraná, Brazil - E-mail: glauciabressan@utfpr.edu.br https://orcid.org/0000-0001-6996-3129

${ }^{4}$ Departamento Acadêmico de Matemática, Universidade Tecnológica Federal do Paraná, Cornélio Procópio, Paraná, Brazil - E-mail: rmolinasouza@utfpr.edu.br https://orcid.org/0000-0002-0638-3650

${ }^{5}$ Departamento Acadêmico de Matemática, Universidade Tecnológica Federal do Paraná, Cornélio Procópio, Paraná, Brazil - E-mail: elenicew@utpfr.edu.br https://orcid.org/0000-0002-8834-4937
} 
Few papers present numerical studies related to the sixth order problem. Numerical solutions are poorly explored, thus we complement this work presenting a numerical study for $(0.1)-(0.2)$ based on Banach's Contraction Principle.

\section{POSITIVE SOLUTIONS}

As presented in [14], we can represent the problem (0.1)-(0.2) as a fixed point of the operator $T: C^{1}[0,1] \rightarrow C^{1}[0,1]$ defined by:

$$
T u(t)=\int_{0}^{1} G(t, s) f(s, u) d s
$$

where $G$ is the Green's function:

$$
\begin{aligned}
G(t, s)=\left(\frac{t^{3}}{2}-\frac{t^{4}}{8}\right) \frac{(1-s)^{4}}{24}+\left(-\frac{t^{3}}{12}+\frac{t^{4}}{16}\right) & \frac{(1-s)^{2}}{2}+\frac{t^{3}}{48}-\frac{5 t^{4}}{192} \\
& +\frac{t^{5}}{120}-\frac{(t-s)^{5}}{120} H(t-s),
\end{aligned}
$$

and

$$
H(\zeta)= \begin{cases}1, & \zeta \geq 0 \\ 0, & \zeta<0\end{cases}
$$

In the sequence, some properties that will be useful related to $G$ are listed.

Propriety 1. How $G(1, s)=\frac{s^{3}}{960}\left(20-25 s+8 s^{2}\right) \geq 0$ following as presented in [14] there are polynomials $p(t)$ and $q(t)$ such that:

$$
p(t) G(1, t) \leq G(t, s) \leq q(t) G(1, s)
$$

where

$$
p(t)=4 t^{2}-4 t+t^{4}, \quad q(t)=\frac{t^{3}}{3}\left(20-25 t+8 t^{2}\right) .
$$

The polynomials $p$ and $q$ are illustrated in Figure 1.

To determine multiple solutions, consider the cone

$$
E=\left\{u \in C^{1}[0,1]: u(0)=0, u(t) \geq 0, \forall t \in[0,1]\right\}
$$

where $C^{1}[0,1]$ is the Banach space of continuously differentiable functions in $[0,1]$ equipped with

$$
\|u\|_{E}=\|u\|_{\infty} .
$$

In order, as $T$ is an integral operator, this is continuous and completely continuous as shown in the proposition (1) 


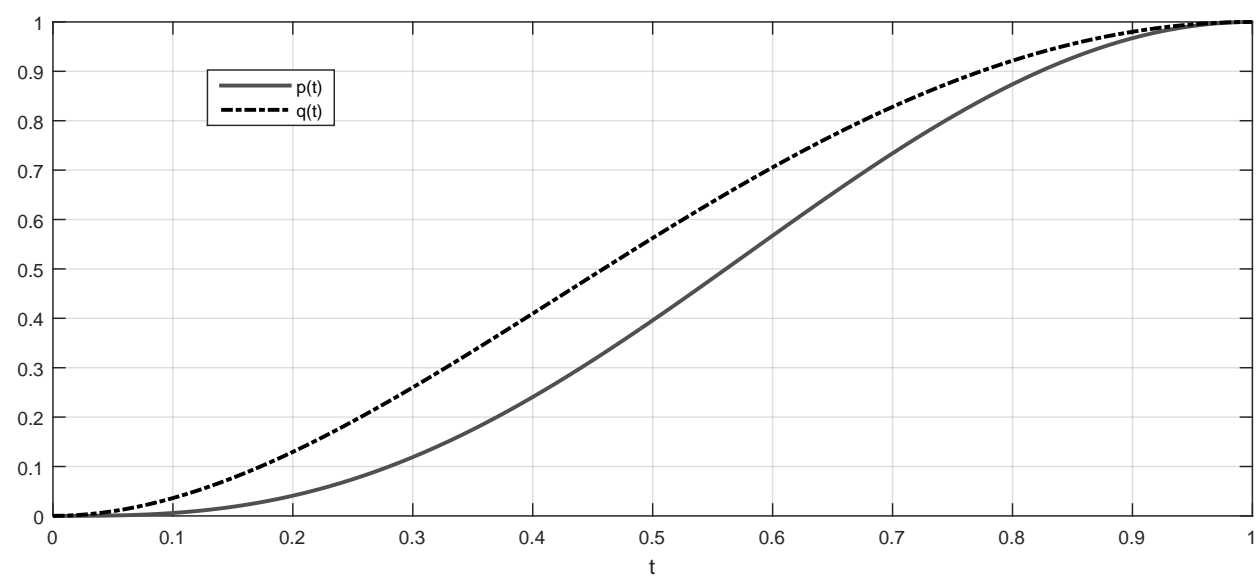

Figure 1: Illustration of polynomials $p$ and $q$ for $t \in[0,1]$.

Proposition 1. The operator $T$ is continuous and completely continuous.

Proof. Continuity follows immediately from the Lebesgue dominated convergence theorem and the fact that

$$
\begin{aligned}
\left|T(u)(t)-T\left(u_{n}\right)(t)\right| & \leq \int_{0}^{1} G(t, s)\left|f(s, u(s))-f\left(s, u_{n}(s)\right)\right| d s, \\
& \leq \int_{0}^{1} G(t, s)\left|f(s, u(s))-f\left(s, u_{n}(s)\right)\right| d s \\
& \leq \int_{0}^{1} q(t) G(1, s)\left|f(s, u(s))-f\left(s, u_{n}(s)\right)\right| d s, \\
& \leq \int_{0}^{1} G(1, s)\left|f(s, u(s))-f\left(s, u_{n}(s)\right)\right| d s,
\end{aligned}
$$

with $u_{n}, u \in E$. To show complete continuity we will use the Arzela-Ascoli's theorem. Let $\Omega \subseteq E$ be bounded, in other words, there exists $\Lambda_{0}>0$ with $\|u\| \leq \Lambda_{0}$ for each $u \in \Omega$. Now if $u \in \Omega$, we have

$$
|(T u)(t)| \leq \int_{0}^{1}|G(t, s)| H_{\Lambda_{0}}(s) d s
$$

where $H_{\Lambda_{0}}$ is determined by the bounded set and function $f$. It is easy to check that $H_{\Lambda_{0}}(s) \in$ $L^{1}[0,1]$. Then imply that $T(\Omega)$ is a bounded equicontinuous family on $[0,1]$. Consequently the Arzela-Ascoli theorem implies $T: E \rightarrow E$ is completely continuous.

To demonstrate the main result of this work, we need to present the main tool to be used.

Avery-Peterson theorem. Now, we need to consider the convex sets

$$
\begin{gathered}
P(\gamma, d)=\{x \in P \mid \gamma(x)<d\} \\
P(\gamma, \alpha, b, d)=\{x \in P \mid b \leq \alpha(x) \text { and } \gamma(x)<d\}
\end{gathered}
$$




$$
P(\gamma, \theta, \alpha, b, c, d)=\{x \in P \mid b \leq \alpha(x), \theta(x) \leq c \text { and } \gamma(x)<d\}
$$

and the closed set

$$
R(\gamma, \psi, a, d)=\{x \in P \mid a \leq \psi(x) \text { and } \gamma(x)<d\}
$$

Theorem 1. Let $P$ be a cone in a real Banach space X. Let $\gamma$ and $\theta$ nonnegative continuous convex functionals on $P, \alpha$ be a nonnegative continuous concave functional on $P$, and $\psi$ be a nonnegative continuous functional on $P$ satisfying $\psi(\lambda x) \leq \lambda \psi(x)$ for $0 \leq \lambda \leq 1$, such that for some positive numbers $\mu$ and $d$,

$$
\alpha(x) \leq \psi(x) \text { and }\|x\| \leq \mu \gamma(x)
$$

for all $x \in \overline{P(\gamma, d)}$. Suppose

$$
T: \overline{P(\gamma, d)} \rightarrow \overline{P(\gamma, d)}
$$

is completely continuous and there exist positive numbers $a, b, c$ with $a<b$, such that

$$
\begin{gathered}
\{u \in P(\gamma, \theta, \alpha, b, c, d) \mid \alpha(u)>b\} \neq \emptyset \text { and } \\
u \in P(\gamma, \theta, \alpha, b, c, d) \Rightarrow \alpha(T u)>b, \\
\alpha(T u)>b \text { for } u \in P(\gamma, \alpha, b, d) \text { with } \theta(T u)>c, \\
0 \notin R(\gamma, \psi, a, d) \text { and } \psi(T u)<a \text { for } \\
u \in R(\gamma, \psi, a, d) \text { with } \psi(u)=a .
\end{gathered}
$$

Then $T$ has at least three distinct fixed points in $\overline{P(\gamma, d)}$.

In order to prove the existence of solutions, we need to consider some basic assumptions.

(H1) For problem (0.1)-(0.2) there is a positive constant $d$ such that:

- For all $(s, v) \in[0,1] \times[0, d]$ then $0 \leq f(s, v) \leq \frac{d}{r_{1}}$

- $r_{1}=\int_{0}^{1} G(1, s) d s$.

The lemma presented will be fundamental for demonstrating our main result.

Lemma 2. Suppose that (H1) holds and $P=E$ and $\gamma()=.\|.\|_{E}$, then $T$ defined in (1.1) fulfills $T: \overline{P(\gamma, d)} \rightarrow \overline{P(\gamma, d)}$. 
Proof. Let us consider $u \in E$ with $\|u\|_{E} \leq d$, so from (H1) we can obtain:

$$
\begin{aligned}
\|T u\|_{E} & =\max _{t \in[0,1]}|(T u)(t)|, \\
& \leq \max _{t \in[0,1]} \int_{0}^{1}|G(t, s) \| f(s, u)| d s \\
& \leq \max _{t \in[0,1]} \int_{0}^{1} q(t) G(1, s)|f(s, u)| d s \\
& \leq \frac{d}{r_{1}}\left[\int_{0}^{1} G(1, s) d s\right] \max _{t \in[0,1]} q(t) \\
& \leq d \max _{t \in[0,1]} q(t) \\
& \leq d .
\end{aligned}
$$

Therefore $T: \overline{P(\gamma, d)} \rightarrow \overline{P(\gamma, d)}$.

Theorem 2 presents conditions under which the problem defined in $(0.1)-(0.2)$ has at least three positive solutions.

Theorem 2. Suppose that the hypothesis (H1) is satisfied. Suppose, in addition, that there exist $a, 0<a<d$ such that $f$ satisfies the following conditions:

(H2) $f(s, u)>\frac{2 a}{r_{2}}, \forall(s, u) \in[0,1] \times[2 a, 8 a]$, where $r_{2}=\frac{423}{2048} \int_{\frac{3}{8}}^{\frac{5}{8}} G(1, s) d s$.

(H3) $f(s, u)<\frac{a}{r_{1}}, \forall(s, u) \in[0,1] \times[0, a]$,

Then, the Problem (0.1)-(0.2) has at least three positive solutions.

Proof. We will apply Avery-Peterson theorem, let us consider $T$ and $P$ as defined before. Furthermore, we need define the following functionals:

$$
\begin{aligned}
\gamma(u) & =\|u\|_{E}, \\
\psi(u) & =\max _{t \in[0,1]}|u(t)|, \\
\theta(u) & =\max _{t \in\left[\frac{3}{8}, \frac{5}{8}\right]}|u(t)| \\
\alpha(u) & =\min _{t \in\left[\frac{3}{8}, \frac{5}{8}\right]}|u(t)| .
\end{aligned}
$$

Therefore, from Lemma 2 we obtain

$$
T: \overline{P(\gamma, d)} \rightarrow \overline{P(\gamma, d)}
$$

and $T$ is completely continuous and there exist positive numbers $b$ and $c$ with $a<b$, such that

$$
\{u \in P(\gamma, \theta, \alpha, b, c, d) \mid \alpha(u)>b\} \neq \emptyset \text { and }
$$




$$
\begin{gathered}
u \in P(\gamma, \theta, \alpha, b, c, d) \Rightarrow \alpha(T u)>b \\
\alpha(T u)>b \text { for } u \in P(\gamma, \alpha, b, d) \text { with } \theta(T u)>c, \\
0 \notin R(\gamma, \psi, a, d) \text { and } \psi(T u)<a \text { for } \\
u \in R(\gamma, \psi, a, d) \text { with } \psi(u)=a .
\end{gathered}
$$

Now, we consider the constants $b$ and $c$ as follows:

$$
b=2 a
$$

and

$$
c=8 a \text {. }
$$

Clearly, we have $\{u \in P(\gamma, \theta, \alpha, b, c, d) \mid \alpha(u)>b\} \neq \emptyset$. Let us demonstrate (1.8).

Using (H2) we obtain

$$
\begin{aligned}
& \alpha(T u)=\min _{t \in\left[\frac{3}{8}, \frac{5}{8}\right]}(T u)(t) \\
&=\min _{t \in\left[\frac{3}{8}, \frac{5}{8}\right]}\left(\int_{0}^{1} G(t, s) f(s, u(s)) d s\right) \\
& \geq \min _{t \in\left[\frac{3}{8}, \frac{5}{8}\right]}\left(\int_{0}^{1} p(t) G(1, s) f(s, u(s)) d s\right) \\
& \geq p(0.375) \int_{0}^{1} G(1, s) f(s, u(s)) d s \\
& \geq \frac{423}{2048} \int_{0}^{1} G(1, s) f(s, u(s)) d s \\
& \geq \frac{423}{2048} \int_{\frac{3}{8}}^{\frac{5}{8}} G(1, s) f(s, u(s)) d s \\
& \geq \frac{423}{2048} \frac{2 a}{r_{2}} \int_{\frac{3}{8}}^{\frac{5}{8}} G(1, s) d s \\
& \geq 2 a=b .
\end{aligned}
$$

Let us demonstrate (1.9). Let $u \in P(\gamma, \alpha, b, d)$ with $\theta(T u)>c$. Then 


$$
\begin{aligned}
& \alpha(T u)=\min _{t \in\left[\frac{3}{8}, \frac{5}{8}\right]}(T u)(t) \\
&=\min _{t \in\left[\frac{3}{8}, \frac{5}{8}\right]}\left(\int_{0}^{1} G(t, s) f(s, u(s)) d s\right) \\
& \geq \min _{t \in\left[\frac{3}{8}, \frac{5}{8}\right]}\left(\int_{0}^{1} p(t) G(1, s) f(s, u(s)) d s\right) \\
& \geq p(0.375)\left(\int_{0}^{1} G(1, s) f(s, u(s)) d s\right) \\
& \geq q(0.625) \frac{p(0.375)}{q(0.625)}\left(\int_{0}^{1} G(1, s) f(s, u(s)) d s\right) \\
& \geq \frac{p(0.375)}{q(0.625)} \max _{t \in\left[\frac{3}{8}, \frac{5}{8}\right]}\left(\int_{0}^{1} q(t) G(1, s) f(s, u(s)) d s\right) \\
& \geq \frac{1}{4} \max _{t \in\left[\frac{3}{8}, \frac{5}{8}\right]}\left(\int_{0}^{1} G(t, s) f(s, u(s)) d s\right) \\
& \geq \frac{1}{4} \theta(T u) \\
&>\frac{1}{4} c=b .
\end{aligned}
$$

Now, to show (1.10) let us consider $u \in R(\gamma, \psi, a, d)$ with $\psi(u)=a$. From (H3) we have,

$$
\begin{aligned}
\psi(\text { Tu }) & =\max _{t \in[0,1]}|(T u)(t)| \\
& \leq \max _{t \in[0,1]} \int_{0}^{1}|G(t, s)||f(s, u)| d s \\
& \leq \max _{t \in[0,1]} \int_{0}^{1} q(t) G(1, s)|f(s, u)| d s \\
& \leq \frac{a}{r_{1}}\left[\int_{0}^{1} G(1, s) d s\right] \max _{t \in[0,1]} q(t) \\
& \leq a .
\end{aligned}
$$

Applying Avery-Peterson theorem we obtain that the problem has at least three distinct solutions in the set $\overline{P(\gamma, d)}$, so these solutions are non-negative. On the other hand, they must satisfy the hypothesis (H2) so they cannot be null. Therefore, the Problem $(0.1)-(0.2)$ has at least three positive.

The example presented below illustrates the hypotheses assumed in Theorem 2 .

Example 1.1. Let us consider (0.1)-(0.2) with

$$
f(t, u)=\left\{\begin{array}{cc}
6 e^{t}+6561+5 \frac{(u-2 a)^{2}}{a} & u \geq 2 a \\
6 e^{t}+\left(\frac{9 u}{2 a}\right)^{4} & u<2 a
\end{array}\right.
$$


Choosing the constants

$$
d=10, a=1,
$$

we can easily verify that in these conditions the hypotheses (H1) and hypotheses of Theorem 2 are satisfied.

\section{NUMERICAL SOLUTIONS}

In this section, we show the existence and uniqueness for (0.1)-(0.2) using Banach Fixed Point Theorem. This approach is classical but very important to define numerical methods for our problem. Let us consider the iterative sequence

$$
\begin{aligned}
u^{k+1}(t) & =\left(T u^{k}\right)(t) \\
& =\int_{0}^{1} G(t, s) f\left(s, u^{k}(s)\right) d s .
\end{aligned}
$$

and the basic assumptions

(H4) $|f(s, u)-f(s, v)| \leq \frac{\beta}{r_{1}}|u(s)-v(s)| ; \quad \forall u, v \in[0, d], s \in[0,1]$ and $\beta \in(0,1)$.

Theorem 3. Suppose that (H1) and (H4) are satisfied. Then (0.1)- (0.2) has an unique solution $u$ with $\|u\|_{E} \leq d$. Moreover, $u^{k+1}=T\left(u^{k}\right) \rightarrow u^{*}$.

Proof. We will prove that the operator $T$ is a contraction. For this, consider $u, v \in E$ with $\|u\|_{E} \leq d$ and $\|v\|_{E} \leq d$. Then

$$
\begin{aligned}
\|T u-T v\|_{E} & =\|(T u-T v)\|_{\infty} \\
& =\max _{t \in[0,1]}\left|\int_{0}^{1} G(t, s)[f(s, u)-f(s, v)] d s\right| \\
& \leq \max _{t \in[0,1]} \int_{0}^{1} G(t, s)|f(s, u)-f(s, v)| d s \\
& \leq \max _{t \in[0,1]} \int_{0}^{1} q(t) G(1, s)|f(s, u)-f(s, v)| d s \\
& \leq\left(\frac{\beta}{r_{1}} \max _{s}|u(s)-v(s)|\right)\left(\max _{t \in[0,1]} q(t)\right) \int_{0}^{1} G(1, s) d s \\
& \leq \beta \max _{s}|u(s)-v(s)| \\
& \leq \beta\|u-v\|_{E} .
\end{aligned}
$$

Therefore, by the principle of contraction there is only one solution that can be obtained as a limit of the sequence $u^{k+1}=T\left(u^{k}\right) \rightarrow u^{*}$.

Motivated by the last result we can define Algorithm 1 . 


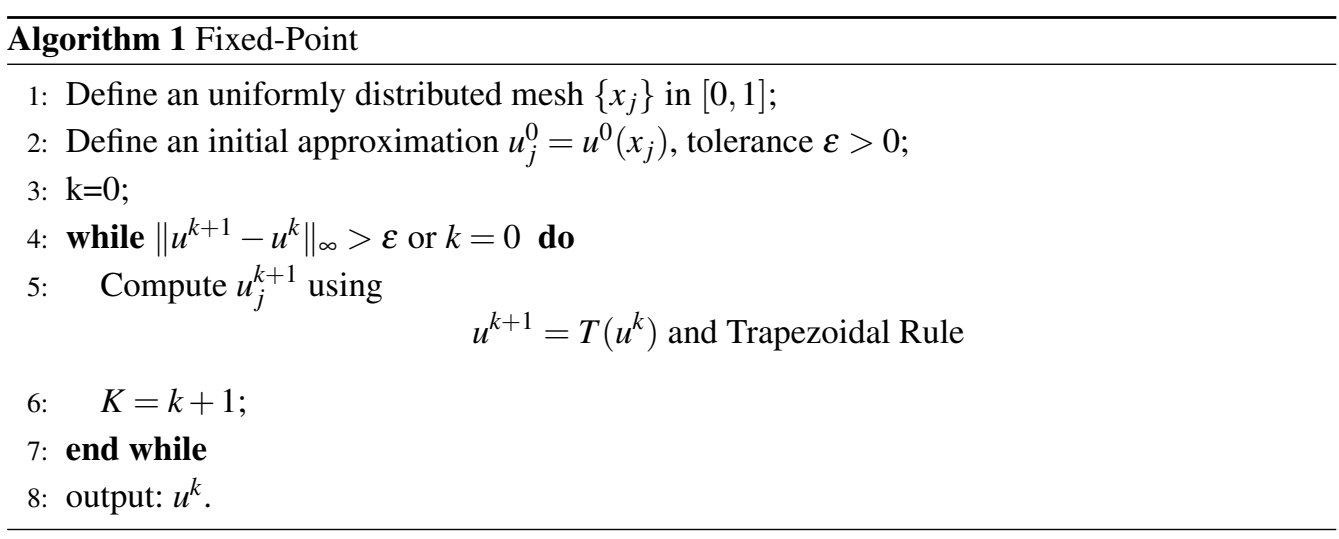

In sequence, examples are presented in order to establish the effectiveness of Algorithm 1. In the Table $1, \varepsilon_{u}^{k}$ denotes $\left\|u^{*}-u^{k}\right\|_{\infty}$ where $u^{*}$ is the exact solution, $\varepsilon^{k}$ denotes $\left\|u^{k+1}-u^{k}\right\|_{\infty}$ and $\bar{\varepsilon}^{k}=\frac{\left\|u^{k+1}-u^{k}\right\|_{\infty}}{\left\|u^{k+1}\right\|_{\infty}}$. Still, "It" denotes "iteration".

Example 2.1. Consider in problem (0.1) - (0.2):

$$
f(t, u)=-\left(32400 t(t-1)^{2}+14400(t-1)^{3}+6480 t^{2}(2 t-2)+720 t^{3}\right) ;
$$

The analytical solution of $(0.1)-(0.2)$ is $u^{*}(t)=t^{3}(1-t)^{6}$. Table 1 contains results of application of the Algorithmic 1 in this example and the results are shown in Figure 2.

Table 1: Algorithm 1 considering Example 2.1.

\begin{tabular}{cccc}
\hline It & $\varepsilon_{u}^{k}$ & $\varepsilon^{k}$ & $\bar{\varepsilon}^{k}$ \\
\hline 1 & $8.015149 \times 10^{-4}$ & 4.746984 & 0.999687 \\
2 & $8.015149 \times 10^{-4}$ & 0 & 0 \\
\hline
\end{tabular}

Figure 2 shows that the solution provided by algorithm 1 is very close to the analytical solution and the error increases when $t$ tends to 1 . This behavior can be justified because in (0.2) does not specify a condition for $u(1)$.

Example 2.2. This example consider the function components of Example 1. We know that, according to theorem 2, the problem of example 1 has at least 3 solutions with a norm less than 1, Algorithm 1 is not the most suitable for determining multiples solutions because it requires that the operator $T$ be in the vicinity of the solution contraction, as seen in Theorem 3. Even so, we performed a test to verify the behavior of Algorithm 1 in an attempt to determine multiple solutions. So inspired by the works [10], [9] and [11], how know that the solutions we are looking for must be continuous and satisfy the condition 0.2. We choose initial approaches that satisfy the conditions $u(0)=u^{\prime}(0)=0$ and $u^{\prime}(1)=0$. Thus, functions parable approaches are reasonable 


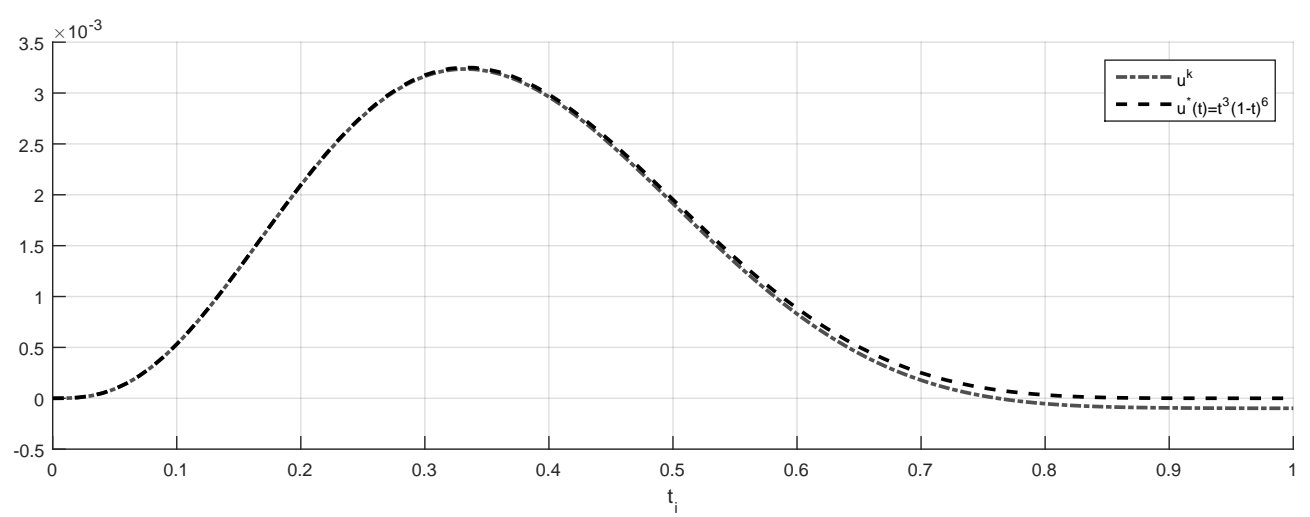

Figure 2: Graph of the analytical solution $u^{k}$ and approximate solution $u^{*}$ obtained by the algorithm 1.

ways to approach the solution. In this sense, our heuristic methodology is to generate parables about starting points as follows:

$$
u^{0}(t)=\zeta\left(2 t^{2}-t^{4}\right)
$$

where the constants $\zeta$ is a random numbers in $[0, d]$. For practical purposes, the proposed procedure is defined by Algorithm 2. It is expected that this procedure returns several solutions.

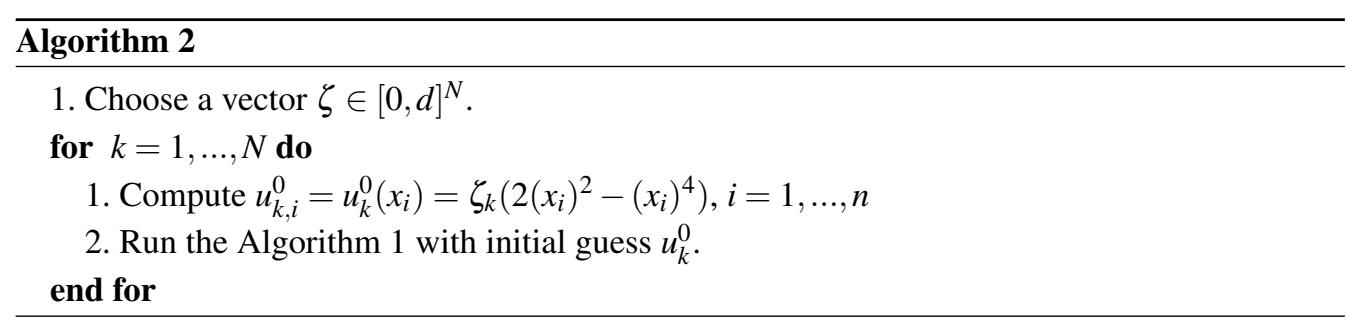

Therefore, it is necessary to establish a way to compare these solutions. Note that the magnitude of the solutions may be different. In this sense, we say that the numeric solutions $u^{*}$ and $u^{* *}$ are equivalent if

$$
\left\|u^{*}-u^{* *}\right\| \leq \max \left\{10^{-4}, 10^{-2} \min \left\{\left\|u^{*}\right\|,\left\|u^{* *}\right\|\right\}\right\} .
$$

is satisfied.

We consider $N=50$ in Algorithm 2 and $\varepsilon=10^{-6}$ in Algorithm 1, we get the convergence of Algorithm 1 in all initializations. Of these 32 initializations converged to the solution $u_{1}^{*}$ the others converged on the $u_{2}^{*}$ solution illustrated in the figure 3 . We can notice that the curves obtained seem to fulfill the hypotheses of Theorem 2 and the conditions (0.2). 

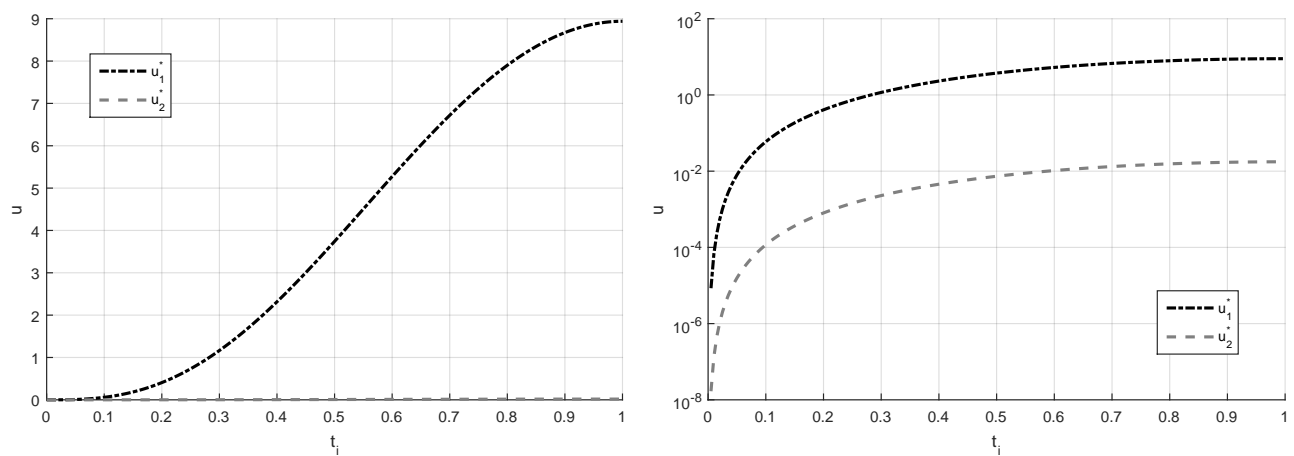

Figure 3: Illustration of solutions obtained for Example 1. The left solutions obtained are illustrated on a linear scale, the right for better visualization we present the solutions on a logarithmic scale

\section{FINAL REMARKS}

This work is restricted to the problem $(0.1),(0.2)$ can have several solutions if the $f$ function meets certain conditions through of the Avery-Peterson theorem. Additionally, conditions are determined for convergence of the interactive sequence $u^{k+1}=T u^{k}$ through the principle of contraction. To complement the analysis, the implementation of this method is performed and nontrivial examples were tested. The results were detailed showing the feasibility of the proposed methods.

RESUMO. Este trabalho apresenta condições para existência de múltiplas soluções para uma equação de sexta ordem com condições de contorno homogêneas usando o teorema de Avery Peterson. Além disso, exemplos não triviais são apresentados e um novo método numérico baseado no Princípio de Contração de Banach é introduzido.

Palavras-chave: soluções numéricas, sexta ordem, problema de valor de contorno e múltiplas soluções.

\section{REFERENCES}

[1] M.M. Adjustovs \& A.J. Lepins. Extremal solutions of a boundary value problem for a sixthorder equation. Differ. Equ., 50(2) (2014), 141-146.

[2] R.P. Agarwal, B. Kovacs \& D. O'Regan. Positive solutions for a sixth-order boundary value problem with four parameters. Bound. Value Probl., 2 (2013), 184-205.

[3] R.P. Agarwal, B. Kovacs \& D. O'Regan. Existence of positive solution for a sixth-order differential system with variable parameters. J. Appl. Math. Comput., 1-2 (2014), 437-454.

[4] J.V. Chaparova, L.A. Peletier, S.A.T.F. Geng \& Y. Ye. Existence and nonexistence of nontrivial solutions of semilinear sixth-order ordinary differential equations. Appl. Math. Lett., 17(10) (2004), $1207-1212$. 
[5] T. Garbuza. On solutions of one 6-th order nonlinear boundary value problem. Math. Model. Anal., 13(3) (2008), 349-355.

[6] K. Ghanbari \& H. Mirzaei. On the isospectral sixth order Sturm-Liouville equation. J. Lie Theory, 23(4) (2013), 921-935.

[7] J.R. Graef \& B. Yang. Boundary value problems for sixth order nonlinear ordinary differential equations. Dynam. Systems Appl., 10(4) (2001), 465-475.

[8] T. Gyulov. Trivial and nontrivial solutions of a boundary value problem for a sixth-order ordinary differential equation. C. R. Acad. Bulgare Sci., 9(58) (2005), 1013-1018.

[9] A.L.M. Martinez, E.V. Castelani, G.M. Bressan \& E.W. Stiegelmeier. Multiple Solutions for an Equation of Kirchhoff Type: Theoretical and Numerical Aspects. Trends in Applied and Computational Mathematics, 19(3) (2018), 559-572.

[10] A.L.M. Martinez, E.V. Castelani \& R. Hoto. Solving a second order m-point boundary value problem. Nonlinear Studies, 26(1) (2018), 15-26.

[11] C.A.P. Martinez, A.L.M. Martinez, G.M. Bressan, E.V. Castelani \& R.M. Souza. Multiple solutions for a fourth order equation with nonlinear boundary conditions: theoretical and numerical aspects. Differential Equations \& Applications, 11(3) (2019), 335-348.

[12] M. Moller \& B. Zinsou. Sixth order differential operators with eigenvalue dependent boundary conditions. Appl. Anal. Discrete Math., 2(7) (2013), 378-389.

[13] G. Suqin, W. Wanyi \& Y. Qiuxia. Dependence of eigenvalues of sixth-order boundary value problems on the boundary. Bull. Aust. Math. Soc., 90(3) (2014), 457-468.

[14] B. Yang. Positive solutions to a nonlinear sixth order boundary value problem. Differential Equations \& Applications, 11(2) (2019), 307-317. 